\title{
Versión en Español del Emotion Regulation Skills Questionnaire: Análisis de su Fiabilidad y Validez
}

\author{
The Spanish Version of the Emotion Regulation Skills Questionnaire: Analysis of \\ Reliability and Validity
}

\author{
Arturo Enrique Orozco-Vargas ${ }^{1}$, Georgina Isabel García-López ${ }^{2}$, Ulises Aguilera-Reyes ${ }^{3}$ y \\ Arturo Venebra-Muñoz ${ }^{4}$
}

\begin{abstract}
Resumen
A pesar de ser un instrumento muy importante, no existe una versión en español del ERSQ que permita el análisis de las estrategias de regulación emocional por medio de esta escala en hispanoparlantes. De esta manera, el objetivo de esta investigación fue analizar las propiedades psicométricas del Emotion Regulation Skills Questionnaire [ERSQ] (consistencia interna del cuestionario, fiabilidad test-retest, validez convergente y discriminante, así como la validez de constructo por medio de un análisis factorial confirmatorio). La muestra se obtuvo de la población general incluyendo un total de 739 adultos mexicanos (453 mujeres y 286 hombres). Los resultados revelaron una consistencia interna $(\alpha=.96)$ y una fiabilidad test-retest (ICC $=0.88)$ muy adecuadas para la escala total. A su vez, se diseñó un modelo de nueve factores el cual se sometió a un análisis factorial confirmatorio encontrándose resultados muy favorables en todos los indicadores $\left(\chi^{2}=752.72, g l=310, \mathrm{CFI}=0.97, \mathrm{TLI}=0.95, \mathrm{SRMR}=0.05, \mathrm{RMSEA}=0.04\right)$. Con base en todos estos resultados, esta investigación confirma las propiedades psicométricas apropiadas del ERSQ.
\end{abstract}

Palabras clave: consistencia interna, fiabilidad test-retest, regulación emocional, validez convergente y discriminante, validez de constructo

\begin{abstract}
Despite being a remarkable instrument, there is not a Spanish version of the ERSQ that allows for the analysis of the strategies of emotion regulation using this scale among Spanish speakers. Therefore, the purpose of this research was to analyze the psychometric properties of the Emotion Regulation Skills Questionnaire [ERSQ] (internal consistency, test-retest reliability, convergent and discriminant validity, as well as construct validity through a confirmatory factor analysis). The sample was collected from the general population including a total of 739 Mexican adults (453 females and 286 males). Results showed a highly suitable internal consistency $(\alpha=.96)$ and a test-retest reliability $(\mathrm{ICC}=0.88)$ for the total scale. In turn, a nine factor model was designed that was analyzed by a confirmatory factor analysis. Significant indicators of model fit were found $\left(\chi^{2}=752.72, d f=310, \mathrm{CFI}=0.97\right.$, TLI $=0.95$, SRMR $=0.05$, RMSEA $\left.=0.04\right)$. Based on all these results, this research confirms the suitable psychometric properties of the ERSQ.
\end{abstract}

Keywords: construct validity, convergent and discriminant validity, emotion regulation, internal consistency, test-retest reliability

\footnotetext{
${ }^{1}$ Doctor en Investigación en Psicología y Educación. Investigador y Profesor de tiempo completo. Universidad Autónoma del Estado de México. Centro Universitario UAEM Atlacomulco; Carretera Toluca-Atlacomulco Km.60, Atlacomulco, México. Correo: dr.enrique.orozco@hotmail.com, aeorozcov@uaemex.mx

${ }^{2}$ Doctora en Investigación en Psicología. Investigador y Profesor de tiempo completo.Universidad Autónoma del Estado de México, México.

${ }^{3}$ Doctor en Medicina Veterinaria. Investigador y Profesor de tiempo completo. Universidad Autónoma del Estado de México, México.

${ }^{4}$ Doctor en Neuroetología. Investigador y Profesor de tiempo completo. Universidad Autónoma del Estado de México, México. 


\section{Introducción}

Las habilidades que regulan las emociones son un elemento indispensable para comprender los procesos que permiten el manejo adecuado de las mismas. Desde los primeros años de vida, los niños comienzan a desarrollar una serie de estrategias por medio de las cuales pueden controlar sus emociones. De manera particular, los padres son los primeros en enseñar a sus hijos diversas acciones encaminadas a la compleja labor de regular sus propias emociones. Una serie de procesos neurofisiológicos, junto con la interacción familiar y social son determinantes en la capacidad que cada persona posee para controlar las emociones dándoles un cauce apropiado (Barrett et al., 2016). Por el impacto tan significativo que tiene en los procesos psicológicos, la regulación emocional ha cobrado una gran importancia en la literatura científica (Salessi \& Omar, 2016).

La regulación emocional ha sido definida como un proceso por medio del cual las distintas emociones que se experimentan en la cotidianidad son manejadas para alcanzar las metas individuales, así como las adaptaciones de orden social y biológico. Este manejo de las emociones implica el entrenamiento, la contención, la evitación, la modulación y la capacidad de mantener las emociones (Eisenberg \& Spinrad, 2004). Desde la década de los 90 's, diversos modelos se han propuesto con la finalidad de dilucidar los mecanismos que convergen en el proceso regulatorio de las emociones.

Uno de los modelos más importantes y con un mayor número de investigaciones llevadas a cabo en todo el mundo fue propuesto por Gross (1998); este incluye cuatro fases (situación, atención, evaluación y respuesta emocional) de las cuales se derivan de manera general una serie de estrategias de regulación emocional: selección de la situación, modificación de la situación, despliegue atencional, cambio cognitivo y modificación de la respuesta. De estas cinco estrategias generales, se han derivado una gran variedad de estrategias de regulación emocional más específicas como lo son la aceptación, conciencia plena, supresión, reevaluación, inhibición, evitación, reenfocamiento, control, rechazo, rumiación y el afrontamiento. Al comparar la inclusión de estas estrategias en la literatura científica, se han identificado la supresión y principalmente la reevaluación cognitiva como las más analizadas en las investigaciones (Zhang et al., 2019). Debido a que pueden modificar la emoción en su totalidad más allá de la pura expresión, las estrategias reevaluativas han demostrado ser las más efectivas y por ende son incluidas constantemente en los estudios que evalúan el manejo de las emociones (Gross \& John, 2003).

Basándose principalmente en el modelo de Gross (1999) y el modelo de regulación emocional basado en el procesamiento emocional propuesto por Hervás (2011), durante las últimas tres décadas diversos autores han desarrollado más de 30 instrumentos diseñados para medir las estrategias de la regulación emocional. Entre los más importantes y usados en investigaciones a nivel nacional e internacional se encuentran: Difficulties in Emotion Regulation Scale (DERS) creado por Gratz y Roemer (2004), Emotion Regulation Questionnaire (ERQ) diseñado por Gross y John (2003), Emotion Regulation Checklist (ERC) desarrollado por Shields y Cicchetti (1997) y el Cognitive Emotion Regulation Questionnaire (CERQ) creado por Garnefski, Kraaij, y Spinhoven (2001).

Por otra parte, con la finalidad de tener un análisis más completo de las diferentes estrategias que son empleadas para regular las emociones, Berking y sus colegas han desarrollado el Modelo de afrontamiento adaptativo de las emociones (Adaptive Coping with Emotions model [ACE]) el cual ha tenido también un impacto muy importante en el diseño de intervenciones psicoterapéuticas (Berking, 2008; Berking \& Schwarz, 2014; Berking \& Whitley, 2014). Este modelo propone que el desarrollo de una serie de habilidades es esencial para conseguir una salud mental adecuada. El propósito de estas habilidades es la transformación de las emociones con miras a lograr las metas personales, así como la aceptación o tolerancia de aquellas emociones que son consideradas como desagradables.

Del Modelo de afrontamiento adaptativo de las emociones ha surgido uno de los instrumentos más importantes empleado a nivel mundial para medir la regulación emocional. El Emotion 
Regulation Skills Questionnaire (ERSQ) es una prueba psicométrica creada por Berking y Znoj (2008). La versión original fue diseñada en alemán con el nombre de Fragebogen zur Selbsteinscha"tzung Emotionaler Kompetenzen. Existen otras dos versiones del ERSQ, una de ellas en inglés (Grant et al., 2018) y la otra en japonés (Fujisato et al., 2017), que han mostrado propiedades psicométricas muy apropiadas confirmando las encontradas en la versión original.

El ERSQ fue diseñado con la finalidad de integrar una serie de habilidades que conforman el proceso adaptativo de la regulación emocional. Estas habilidades permiten (a) experimentar las emociones de una manera consciente, (b) identificar los efectos que tienen las emociones en el organismo, (c) otorgarle un nombre a cada una de las emociones que se perciben, (d) comprender aquello que origina una emoción, (e) tener la capacidad para aceptar las emociones sin querer modificarlas, (f) tolerar el impacto de las emociones negativas, $(\mathrm{g})$ enfrentarse a situaciones que conllevan emociones negativas, (h) animarse a sí mismo durante situaciones de angustia emocional, y (h) llevar a cabo diferentes acciones con el objetivo de cambiar los efectos de las emociones negativas.

A diferencia de las otras escalas que miden la regulación emocional, una de las principales características del ERSQ es que mide de manera exhaustiva el proceso intraindividual del manejo de las emociones. Particularmente, esta escala integra las estrategias de regulación emocional adaptativas (e.g., consciencia plena, aceptación, disposición a confrontar y claridad) a través de las cuales se puede conseguir un control efectivo de las experiencias emocionales. Asimismo, de forma interna, cada persona tiene la posibilidad de regular algún componente emocional que identifique previo a la generación de la emoción que se está activando o también lo puede hacer en los componentes que se derivan de la experiencia emocional. Por ejemplo, sabiendo anticipadamente que la ruptura de una relación romántica es inminente, los miembros de la pareja pueden comenzar a aceptar las consecuencias emocionales que traerá esa disolución. Por otra parte, con respecto a reacción posterior al evento emocional, tiempo después de haber terminado esa relación, cada uno puedo tomar conciencia de las emociones negativas que experimentaron cuando estuvieron juntos.

Otra ventaja que tiene, en comparación con otras escalas que tienen pocas dimensiones, es que el ERSQ tiene nueve dimensiones (Consciencia emocional, Sensación, Claridad, Conocimiento, Aceptación, Tolerancia, Auto-Ayuda, Disposición a confrontar y Modificación) por medio de las cuales se miden estrategias cognitivas y conductuales. Por otra parte, el ERSQ ha sido empleado para investigar la regulación emocional tanto en población en general como en población clínica permitiendo identificar importantes diferencias. De esta manera, ha sido posible documentar los efectos de la regulación emocional en una gran variedad de procesos psicológicos entre los que destacan el trastorno de déficit de atención e hiperactividad y el trastorno límite de la personalidad (Cavelti et al., 2019), psicosis (Lawlor et al., 2020), depresión y ansiedad (Lukas et al., 2018), calidad de vida y rasgos de personalidad (Pocnet et al., 2017), apego (Owens et al., 2018), pérdida de control e impotencia (Berking et al., 2010), imagen corporal (Shriver et al., 2021), violencia de pareja (Berke et al., 2019), entre otros.

Específicamente, el ERSQ ha sido usado ampliamente alrededor del mundo para analizar el impacto de las estrategias de regulación emocional en la depresión y la autocompasión (Diedrich et al., 2017), la procrastinación (Eckert et al., 2016), el estrés (Lincoln et al., 2015), el afecto positivo y negativo (Berking et al., 2008), la autoaceptación, las relaciones positivas con los otros, el propósito en la vida, el crecimiento personal, el control en el ambiente y la autonomía (Moradi-Siahafshadi et al., 2018), entre muchas otras más investigaciones. A pesar de su importancia, no existe una versión en español del ERSQ que permita el análisis de este constructo en población de habla hispana. Con la finalidad de llenar este vacío en la literatura, los objetivos de esta investigación fueron: (a) confirmar la estructura de nueve factores del Emotion Regulation Skills Questionnaire en una muestra de 739 participantes; (b) estimar la consistencia interna del cuestionario y la fiabilidad test-retest; (c) analizar la validez convergente y discriminante del ERQS; (d) examinar la validez de constructo 
de la escala por medio de un análisis factorial confirmatorio.

\section{Método}

\section{Participantes}

La recolección de datos se llevó a cabo en ocho dependencias públicas y siete empresas particulares. La muestra estuvo conformada por un total de 739 trabajadores mexicanos de los cuales 453 fueron mujeres $(61.3 \%)$ y 286 hombres (38.7\%). El promedio de edad fue de 40.3 años $(D E=12.4)$. Con respecto a su nivel educativo, el $2.7 \%$ de los participantes tenía la primaria concluida, el $7.4 \%$ concluyó la secundaria, un $37.5 \%$ tenía la preparatoria, el $48.2 \%$ terminó estudios de licenciatura y solamente el $4.2 \%$ tenía estudios de posgrado. Asimismo, el 37.0\% estaba soltero, el $45.3 \%$ estaba casado, el $15.3 \%$ se había divorciado o separado y el $2.4 \%$ era viudo. El $42.5 \%$ no tenía hijos, el $17.5 \%$ tenía un hijo, el $28.4 \%$ tenía dos hijos, el $8.2 \%$ tenía 3 hijos y $3.4 \%$ tenía más de 3 hijos.

\section{Instrumentos}

El Cuestionario de Habilidades de Regulación Emocional (Emotion-Regulation Skills Questionnaire [ERSQ]) fue desarrollado en su versión original en alemán por Berking y Znoj (2008). Para llevar a cabo el análisis psicométrico de las propiedades de este cuestionario, se usó la versión en inglés propuesta por Grant et al. (2018). El ERSQ es un instrumento compuesto por 27 reactivos que utiliza un formato de respuesta tipo Likert de 5 puntos oscilando entre 0 (no del todo) y 4 (casi siempre). Los reactivos están agrupados en nueve factores. El primer factor, llamado de la consciencia emocional, mide el grado con el cual las personas tienen la capacidad de identificar y tomar consciencia de las emociones que experimentan. El segundo factor, denominado Sensación, representa la capacidad que tienen las personas para saber que sus emociones les pueden ayudar a identificar la manera en que ellos se están sintiendo. La tercera dimensión de Claridad mide el nivel de claridad con el cual cada persona identifica el tipo de emoción que está experimentando. Por otra parte, los reactivos agrupados en el factor de
Conocimiento describen la capacidad que tienen las personas para comprender por qué se sienten de tal manera en cada una de las situaciones que han vivido recientemente. La siguiente dimensión, denominada Aceptación, mide el nivel de aceptación que se tiene frente a cada una de las emociones que se experimentan. Por medio del sexto factor, denominado Tolerancia, se puede identificar qué tan tolerantes son las personas cuando están en una situación emocional. A su vez, los reactivos agrupados en la Disposición a confrontar miden la capacidad que tienen las personas para conseguir sus propias metas a pesar del tipo de sentimientos que estén experimentando. El octavo factor denominado Auto-Ayuda se refiere a todo aquello que hacen las personas para animarse a sí mismos cuando sienten distintas emociones. Finalmente, el factor llamado Modificación mide la manera en que las personas pueden influenciar sus sentimientos.

$\mathrm{Al}$ responder la escala, los participantes deben evaluar las afirmaciones que se incluyen en cada uno de los reactivos considerando si ocurrieron "La semana pasada..." Con respecto a las propiedades psicométricas del ERSQ, se encontró que el instrumento posee una consistencia interna muy adecuada (alfa de Cronbach de 0.96 para la escala total). De la misma manera, cada una de las dimensiones obtuvo buenos indicadores (Consciencia emocional $\alpha=.76$, Sensación $\alpha=.75$, Claridad $\alpha=.85$, Conocimiento $\alpha=.85$, Aceptación $\alpha=.79$, Tolerancia $\alpha=.88$, Disposición a confrontar $\alpha=.77$, Auto-Ayuda $\alpha=.73$ y Modificación $\alpha=.74$ ) (Grant et al., 2018). Por otra parte, la fiabilidad test-retest mostró índices adecuados $\left(r_{t t}=.75\right.$ para la escala total y de 0.48 a 0.74 para las nueve dimensiones). De la misma manera, los resultados de diversos análisis factoriales exploratorios y confirmatorios fueron apropiados proporcionando evidencias de la estructura multidimensional del ERSQ (Berking et al., 2008; Berking \& Znoj, 2008; Fujisato et al., 2017).

El Cuestionario cognitivo de regulación emocional (Cognitive Emotion Regulation Questionnaire [CERQ]) fue diseñado por Garnefski et al. (2001). Este cuestionario cuenta con 36 reactivos que miden los aspectos cognitivos involucrados en la regulación emocional. El CERQ puede ser administrado en la población en general, así como en el ámbito 
clínico. En la presente investigación se empleó la versión en español propuesta por DomínguezSánchez et al. (2013). Además de medir el estilo cognitivo general, también evalúa las estrategias emocionales que emplean las personas después de vivir un evento en concreto. Esta escala cuenta con nueve dimensiones incluyendo cuatro reactivos en cada una. Las posibles respuestas se ofrecen en una escala tipo Likert de 5 opciones oscilando entre el 1 (casi nunca) y el 5 (casi siempre). Las puntuaciones altas reflejan un mayor uso de cada una de las nueve estrategias de regulación emocional. El cuestionario ha mostrado características psicométricas adecuadas. Específicamente, la consistencia interna ha oscilado entre .68 y .93 (Garnefski \& Kraaij, 2007). En la presente investigación encontramos índices similares desde 0.65 para Aceptación, .69 para Autoculpa, .75 para Rumiación, .80 para Reenfoque positivo, 0.81 para Reenfoque en la planeación, .84 para Reevaluación positiva, .88 para Poner en perspectiva, .90 para Catastrofización, hasta .91 para Culpar a otros. Asimismo, la validez convergente y discriminante también ha sido adecuada la cual ha sido confirmada mediante las relaciones significativas entre el cuestionario y otras variables como la depresión y la ansiedad (Garnefski \& Kraaij, 2007).

La Escala de dificultades en la regulación emocional (Difficulties in Emotion Regulation Scale [DERS] fue creada por Gratz y Roemer (2004). La escala incluye 36 reactivos que evalúan una serie de dificultades en la regulación emocional enfatizando las emociones negativas. Los reactivos son agrupados en seis dimensiones incluyendo entre cinco y seis reactivos con excepción de una dimensión que contiene ocho reactivos. En este estudio se empleó la versión en español propuesta por Medrano et al. (2013). El instrumento se contesta por medio de una escala tipo Likert de 5 opciones de respuesta que oscilan entre 1 (casi nunca) y 5 (casi siempre). Debido a la naturaleza de la escala, las puntuaciones más altas señalan niveles más altos de desregulación emocional. La consistencia interna de la escala ha mostrado ser apropiada tanto para el puntaje total $(\alpha=.94)$, así como para las seis dimensiones (valores de alfa oscilando entre .80 y .91). En este estudio los coeficientes alfa de Cronbach fueron satisfactorios, Dificultades en el control de impulsos $(\alpha=.82)$, Acceso limitado a estrategias de regulación $(\alpha=.92)$, Falta de aceptación emocional $(\alpha=.87)$, Interferencia en conductas dirigidas a metas $(\alpha=.79)$, Falta de conciencia emocional $(\alpha=.88)$ y Falta de claridad emocional $(\alpha=.75)$. En cuanto a su validez, el análisis factorial exploratorio identificó los seis factores que explicaron el $55.68 \%$ del total de la varianza de las variables medidas (Gratz \& Roemer, 2004).

La lista de verificación de síntomas 90 (Symptom Checklist-90-Revised [SCL-90-R]) creada por Derogatis (1994) mide el nivel de distrés psicológico que experimenta una persona. Esta escala puede ser empleada en población general, así como en personas que padezcan trastornos mentales y físicos. Está conformada por 90 reactivos agrupados en nueve factores (Somatización, Obsesivo compulsivo, Sensitividad interpersonal, Depresión, Ansiedad, Hostilidad, Ansiedad fóbica, Ideación paranoide y Psicoticismo. De la misma manera, se puede reportar un Índice de severidad global). En una escala tipo Likert de 5 opciones, los participantes evalúan el grado de distrés que han sentido durante los últimos siete días con respecto a la lista de los 90 síntomas. Este instrumento ha reportado propiedades psicométricas de validez y confiabilidad muy aceptables (Schmitz et al., 1999) mostrando una consistencia interna que ha oscilado entre .77 y .90 (Derogatis, 1994). En este estudio se encontraron coeficientes alfa de Cronbach desde .72 para Hostilidad, .78 para Ideación paranoide, 0.81 para Ansiedad fóbica, 0.84 para Sensitividad interpersonal, .85 para Psicoticismo, .88 para Obsesivo compulsivo, .88 para Ansiedad, .90 para Somatización, hasta .92 para Depresión. Para la presente investigación, se usó la versión adaptada y traducida al español de Cruz-Fuentes et al. (2005).

\section{Procedimiento}

Después de haber localizado las posibles dependencias públicas y las empresas particulares necesarias para conseguir la muestra que se había determinado, se realizaron diversas gestiones en cada una de ellas para obtener la autorización correspondiente. Una vez que se permitió la recolección de datos, se hizo la invitación en general a los trabajadores de cada organización 
Tabla 1. Media, desviación estándar y consistencia interna

\begin{tabular}{|c|c|c|c|c|c|c|c|c|}
\hline Factor & Media & $\mathrm{DE}$ & $\begin{array}{c}\text { Media } \\
\text { Mujeres }\end{array}$ & $\begin{array}{c}\text { DE } \\
\text { Mujeres }\end{array}$ & $\begin{array}{c}\text { Media } \\
\text { Hombres }\end{array}$ & $\begin{array}{c}\mathrm{DE} \\
\text { Hombres }\end{array}$ & $\alpha$ & $\begin{array}{l}\text { omega de } \\
\text { McDonald }\end{array}$ \\
\hline Consciencia & 3.17 & .58 & 3.28 & .49 & 3.01 & .64 & .71 & .75 \\
\hline Sensación & 2.37 & .59 & 2.41 & .54 & 2.35 & .58 & .83 & .84 \\
\hline Claridad & 2.73 & .72 & 2.91 & 69 & 2.53 & .77 & .88 & .88 \\
\hline Conocimiento & 2.28 & .47 & 2.25 & .52 & 2.31 & .38 & .75 & .73 \\
\hline Aceptación & 3.09 & .73 & 3.40 & .61 & 2.88 & .57 & .92 & .93 \\
\hline Tolerancia & 2.37 & .58 & 2.31 & .47 & 2.38 & .70 & .78 & .80 \\
\hline Disp. Conf. & 2.38 & .90 & 2.70 & .75 & 2.11 & .59 & .64 & .65 \\
\hline Auto-ayuda & 2.74 & .74 & 2.51 & .51 & 2.97 & .64 & .83 & .82 \\
\hline Modificación & 2.48 & .82 & 2.45 & .71 & 2.52 & .76 & .80 & .80 \\
\hline Punt. total & 2.70 & .72 & 3.03 & .58 & 2.58 & .69 & .94 & .94 \\
\hline
\end{tabular}

para participar en una investigación. A quienes decidieron participar de manera voluntaria en ella se les asignó un día y una hora durante la cual estarían respondiendo los cuatro instrumentos. Cada organización proporcionó un salón donde los trabajadores contestaron las escalas en pequeños grupos que oscilaron entre 3 y 10 personas. La recolección de datos se hizo siguiendo todas las normas éticas que se han establecido en México para llevar a cabo una investigación con seres humanos. Antes de participar en el estudio, se leyó con los trabajadores el consentimiento informado y se respondieron las preguntas que tuvieran con respecto a las características generales de la investigación. Aquellos que desearon participar en el estudio contestaron los instrumentos en un promedio de 40 minutos.

Con respecto a la traducción al español y la adaptación a la población mexicana del ERSQ, en una primera etapa, tres investigadores que son bilingües tradujeron al español los reactivos de la versión en inglés del cuestionario. Una vez que se contó con estas tres versiones, se discutieron todas las discrepancias con la finalidad de obtener una versión final hasta que se llegó a un consenso. La segunda etapa consistió en la traducción del español al inglés por parte de un traductor profesional. Al tener esa versión en inglés, se volvieron a discutir todas las discrepancias entre esta versión y la versión original. Se hicieron los cambios pertinentes en las cuatro únicas diferencias que se encontraron y finalmente se hizo un ajuste a la versión final considerando los aspectos culturales de la población mexicana a la cual se iba a aplicar el instrumento. Con la finalidad de asegurar la exactitud de esta adaptación, en una tercera etapa se llevó a cabo un estudio piloto con 12 trabajadores de una empresa particular y 15 de una organización pública. En este estudio piloto se analizaron la redacción y la compresión que tuvieron los participantes de cada uno de los reactivos, así como posibles confusiones en la interpretación de las palabras. Las dudas que surgieron se discutieron y se hicieron los ajustes apropiados. De esta manera se concluyó todo el proceso confirmando la versión en español que se presenta en este artículo.

\section{Análisis de datos}

En un primer momento se analizaron los coeficientes alfa, omega de McDonald y las correlaciones bivariadas entre las dimensiones del ERSQ. Para el cálculo del valor de omega se usó el programa estadístico $\mathrm{R}$, versión 3.2.1 (R Core Team, 2020). Posteriormente, se analizaron la consistencia interna del cuestionario, la fiabilidad test-retest y las correlaciones entre las puntuaciones de las subescalas del ERSQ, así como la validez convergente y discriminante del cuestionario. En un tercer momento se llevó a cabo un análisis factorial confirmatorio con el propósito de validar la estructura del ERSQ. Este análisis se llevó a cabo con el programa LISREL 8.8 (Jöreskog \& Sörbom, 1996) usando el procedimiento de máxima probabilidad. Con la finalidad de evaluar la bondad del ajuste de los datos se incluyeron diversos criterios (Comparative Fit Index, TuckerLewis Index, Standardized Root Mean Square Residual, Root Mean Square Error Aproximation). Siguiendo la propuesta de Hu y Bentler (1999) y de Schumacker y Lomax (2016) valores iguales o superiores a .95 para el CFI y el TLI son indicativos de un buen ajuste, valores inferiores a .08 del SRMR y cercanos a .06 del RMSEA indican un buen ajuste. 


\section{Resultados}

\section{Fiabilidad}

Siguiendo los criterios propuestos por George y Mallery (2013) y Flora (2020) se calcularon el alfa de Cronbach y el omega de McDonald con la finalidad de determinar la consistencia interna de los reactivos del ERSQ (ver Tabla 1).

\section{Fiabilidad test-retest}

Con el objetivo de analizar la fiabilidad testretest del ERSQ, se llevó a cabo una segunda medición cuatro semanas después de haber realizado la primera recolección de datos. De los 739 trabajadores que respondieron originalmente el instrumento, 284 mostraron su interés por volver a contestar el cuestionario. Este análisis se llevó a cabo calculando los coeficientes de correlación intraclase de las puntuaciones del ERSQ en la primera y segunda administración (Koo \& Li, 2016; Shrout \& Fleiss, 1979). Los resultados indicaron que las puntuaciones totales del Cuestionario tuvieron una fiabilidad test-retest muy buena durante el periodo que osciló entre la administración original y la que se llevó a cabo cuatro semanas después (ICC=.88). La fiabilidad test-retest para cada uno de los factores también fue adecuada y significativa $(p<.001)$, Consciencia emocional ICC $=.69$, Sensación ICC $=.71$, Claridad ICC $=.80, \quad$ Conocimiento ICC $=.72$, Aceptación ICC $=.65$, Tolerancia ICC $=.81$, Disposición a confrontar ICC $=.73$, Auto-Ayuda ICC=.87 y Modificación ICC=.70).

\section{Análisis preliminares}

Con el objetivo de proporcionar datos preliminares de las propiedades psicométricas del ERSQ, correlaciones entre las puntuaciones de las subescalas del ERSQ fueron calculadas. Como se muestra en la Tabla 2, todas las correlaciones entre las subescalas y la puntuación total del ERSQ se presentaron en las direcciones que se esperaban y fueron estadísticamente significativas. Los resultados revelaron que las correlaciones oscilaron entre .40 (entre Tolerancia y Auto-Ayuda) y .91 (entre Conciencia emocional y Aceptación).

$\mathrm{Al}$ analizar las relaciones entre la edad y las subescalas del ERSQ y del SCL-90-R, en términos generales entre mayor edad tuvieron los participantes mejor fue su regulación emocional, así como mayor fue la presencia de distrés psicológico. Específicamente, las correlaciones con mayor magnitud se encontraron entre la edad y la conciencia emocional, la edad y la aceptación, así como la edad y la puntación total del ERSQ. De la misma manera, se encontraron correlaciones positivas entre la edad y la ansiedad, la edad y los síntomas obsesivo-compulsivos, así como la edad y la puntuación total del SCL-90-R. Por otra parte, las correlaciones significativas de mayor magnitud entre las subescalas del ERSQ y del SCL-90-R fueron todas negativas y se encontraron entre la conciencia y la hostilidad, la sensación y la depresión, la claridad y la sensitividad interpersonal, el conocimiento y la ansiedad, la aceptación y la depresión, la disposición a confrontar y la hostilidad, la auto-ayuda y la depresión, así como entre la modificación y la ansiedad (ver Tabla 2).

Por otra parte, debido a las diferencias entre mujeres y hombres que se han documentado en estudios previos, se empleó la prueba $t$ de Student con el propósito de analizar las posibles diferencias que pudiéramos encontrar en las 33 dimensiones que conforman los cuatro instrumentos que se incluyeron en esta investigación. Con respecto a los factores que conforman el ERSQ se encontraron diferencias estadísticamente significativas entre mujeres y hombres donde las mujeres reportaron un nivel más alto en la Conciencia emocional $(t$ $(737)=2.29 ; p=.020)$, la Claridad $(t(737)=3.10$; $p=.013)$, la Aceptación $(t(737)=4.24 ; p=.001)$, la Disposición a confrontar $(t(737)=1.43 ; p=.042) \mathrm{y}$ la Puntuación total $(t \quad(737)=3.94 ; \quad p=.005)$ mientras que los hombres presentaron un nivel más alto en la Auto-ayuda ( $t \quad(737)=-3.19$; $p=.028)$. Con respecto al instrumento CERQ, se identificaron diferencias significativas en las cuales las mujeres tuvieron un nivel más alto en la Aceptación $(t$ (737) $=1.94 ; p=.033)$, la Rumiación $(t(737)=4.19 ; p=.002)$ y el Reenfoque en la planeación $(t(737)=3.29 ; p=.020)$, mientras que los hombres tuvieron un nivel más alto en el Reenfoque positivo $(t$ (737)=-5.33; $p=.004)$. Al analizar las diferencias significativas en el instrumento DERS, se identificó que mientras los hombres tuvieron un nivel más alto en la Falta de conciencia emocional $(t(737)=-3.03 ; p=.018)$, las 
Tabla 2. Correlaciones entre la edad, las dimensiones del ERSQ y las dimensiones

del SCL-90-R

\begin{tabular}{|c|c|c|c|c|c|c|c|c|c|c|c|c|c|c|c|c|c|c|c|c|}
\hline & 1 & 2 & 3 & 4 & 5 & 6 & 7 & 8 & 9 & 10 & 11 & 12 & 13 & 14 & 15 & 16 & 17 & 18 & 19 & 2021 \\
\hline 1.Edad & -- & & & & & & & & & & & & & & & & & & & \\
\hline 2.Conc. Emo. & $.28 * *$ & -- & & & & & & & & & & & & & & & & & & \\
\hline 3.Sensación & .11 & $.73 * *$ & -- & & & & & & & & & & & & & & & & & \\
\hline 4.Claridad & $.15^{*}$ & $.84 * *$ & $.73 * *$ & -- & & & & & & & & & & & & & & & & \\
\hline 5.Conocimiento & .13 & $.72 * *$ & $.63 * *$ & $.65 * *$ & -- & & & & & & & & & & & & & & & \\
\hline 6.Aceptación & $.21 *$ & $.91 * *$ & $.85^{* *}$ & $.72 * *$ & $.72 * *$ & -- & & & & & & & & & & & & & & \\
\hline 7.Tolerancia & .08 & $.84 * *$ & $.78 * *$ & $.84 * *$ & $.64 * *$ & $.72 * *$ & -- & & & & & & & & & & & & & \\
\hline 8.Disp. Conf. & $.20 *$ & $.88 * *$ & $.51 * *$ & $.59 * *$ & $.39 *$ & $.58 * *$ & $.57 * *$ & -- & & & & & & & & & & & & \\
\hline 9.Auto-Ayuda & .11 & $.74 * *$ & $.71 * *$ & $.70 * *$ & $.79 * *$ & $.85^{* *}$ & $.40 * *$ & $.63 * *$ & -- & & & & & & & & & & & \\
\hline 10.Modificación & $.18 *$ & $.83 * *$ & $.42 * *$ & $.58 * *$ & $.58 * *$ & $.68 * *$ & $.71 * *$ & $.74 * *$ & $.75^{* *}$ & -- & & & & & & & & & & \\
\hline 11.ERQS total & $.32 * *$ & $.79 * *$ & $.69 * *$ & $.77 * *$ & $.70 * *$ & $.73 * *$ & $.68 * *$ & $.82 * *$ & $.85^{* *}$ & $.88^{* *}$ & -- & & & & & & & & & \\
\hline 12.Somatización & .05 & -.10 & -.08 & -.09 & $-.17 *$ & -.04 & -.03 & -.05 & -.08 & -.11 & $-.15^{*}$ & -- & & & & & & & & \\
\hline 13.Obs-Comp & $.18^{*}$ & .05 & -.11 & -.05 & -.07 & -.02 & -.04 & $-.14^{*}$ & -.04 & -.05 & $-.13^{*}$ & $.15^{*}$ & -- & & & & & & & \\
\hline 14.Sens. Interp. & .06 & -.14 & -.03 & $-.23 * *$ & $-.21 * *$ & -.10 & -.02 & -.07 & $-.13 *$ & $-.14 *$ & -.08 & .09 & $.14 *$ & -- & & & & & & \\
\hline 15.Depresión & .09 & -.09 & $-.15 *$ & $-.14 *$ & $-.18 *$ & $-.18 * *$ & -.08 & $-.17 *$ & $-.22 * *$ & $-.18 *$ & $-.18 *$ & $.18 * *$ & .11 & $.21 * *$ & -- & & & & & \\
\hline 16.Ansiedad & $.18^{*}$ & $-.17 *$ & -.04 & -.08 & $-.28 * *$ & -.08 & -.01 & $-.14^{*}$ & $-.15^{*}$ & $-.25 * *$ & $-.21 * *$. & $.21 * *$ & $.23 * *$ & $.16^{*}$ & $.21 * *$ & -- & & & & \\
\hline 17.Hostilidad & $.14^{*}$ & $-.25 * *$ & -.02 & -.05 & $-.15^{*}$ & -.07 & -.11 & $-.23 * *$ & -.11 & $-.17 *$ & $-.24 * *$ & $.14^{*}$ & $.19^{* *}$ & $.21 * *$ & $.14 *$ & $.21 * *$ & -- & & & \\
\hline 18.Ans. Fóbica & .02 & -.08 & .03 & -.03 & -.04 & -.01 & -.03 & $-.13 *$ & -.03 & -.11 & $-.16^{*}$ & $.18 * *$ & $.13 *$ & $.17 * *$ & $.18 * *$ & $.25 * *$ & $.15^{*}$ & -- & & \\
\hline 19.Ideac. Paran. & .08 & .02 & $-.14 *$ & -.06 & $-.14 *$ & -.02 & -.05 & -.04 & -.04 & -.08 & -.11 & $.16^{*}$ & $.17 * *$ & $.20 * *$ & $.24 * *$ & $.13 *$ & $.20 * *$ & $.17 *$ & -- & \\
\hline 20.Psicoticismo & .03 & -.04 & -.04 & -.02 & -.03 & .01 & .07 & -.02 & .01 & -.05 & -.08 & $.13^{*}$ & .11 & $.14 *$ & $.22 * *$ & $.18 * *$ & $.14^{*}$ & $.13^{*}$ & $.15^{*}$ & -- \\
\hline $\begin{array}{l}\text { 21.SCL-90-R } \\
\text { total } \\
\end{array}$ & $.21 * *$ & $-.18 *$ & -.03 & -.07 & $-.20 * *$ & -.11 & -.04 & $-.18^{* * *}$ & $-.19 * *$ & $-.27 * *$ & $-.19 * *$ & $.21 * *$ & $.18^{* *}$ & $.27 * *$ & $.30 * *$ & $.32 * *$ & $.25^{* *}$ & $.18^{* *}$ & $.20 * *$ & $.17 *--$ \\
\hline
\end{tabular}

mujeres tuvieron un mayor nivel de Interferencia en conductas dirigidas a metas $(t(737)=1.94$; $p=.027)$ y en las Dificultades en el control de impulsos $(t$ (737) $=5.24 ; p=.010)$. Con respecto al nivel de distrés psicológico medido con la lista de verificación de síntomas 90 , se presentaron diferencias significativas en cuatro dimensiones donde los hombres tuvieron mayores niveles de Hostilidad $(t(737)=-5.18 ; p=.028)$ y los síntomas Obsesivo-compulsivos $(t$ (737) $=-2.84 ; p=.001)$, mientras que las mujeres tuvieron un nivel más alto de Somatización $(t(737)=1.53 ; p=.031)$ y Depresión $(t(737)=3.24 ; p=.015)$.

\section{Validez discriminante y convergente}

En una etapa posterior se analizaron la validez convergente y discriminante del ERQS. En un primer momento se llevaron a cabo tres procedimientos con el objetivo de verificar la validez discriminante del instrumento. Como se muestra en la Tabla 3, se realizó un análisis de correlaciones de Pearson entre las puntuaciones del ERQS y el nivel de distrés psicológico (medido por el SCL-90-R). Estas correlaciones tuvieron una menor magnitud en comparación con las correlaciones entre el ERQS y el Cuestionario cognitivo de regulación emocional, así como con la Escala de dificultades en la regulación emocional que se emplearon para verificar la validez convergente. Posteriormente se calcularon los valores de la varianza media extraída (AVE) y las raíces cuadradas del AVE (Ver Tabla 3). Los resultados mostraron que el valor del AVE se ubicó por arriba de .50 que es considerado el mínimo requerido (Fornell \& Larcker, 1981). Asimismo, las raíces cuadradas del AVE fueron mayores a la correlación entre todos los factores que se analizaron (Netemeyer et al., 1990). Finalmente, se calculó la Proporción HeterorasgoMonorasgo (Heterotrait-Monotrait Ratio [HTMT]). La proporción HTMT ha sido definida como la media de las correlaciones entre los factores que miden distintos constructos en relación con el promedio de las correlaciones de los factores de un mismo constructo (Henseler et al., 2015). De acuerdo con Kline (2011), los valores inferiores a .85 evidencian una validez discriminante adecuada. Los resultados mostraron que el valor de la proporción HTMT existente entre las correlaciones de los factores osciló entre .22 (Tolerancia-Puntuación total del SCL-90-R) y .78 (Aceptación-Puntuación total del DERS) corroborando que en todos los casos la proporción HTMT fue inferior a .85 . Los resultados de estos tres procedimientos en conjunto sugieren evidencia de la validez discriminante del ERQS.

Con respecto a la validez convergente, se emplearon dos de las escalas más importantes a nivel internacional para medir la regulación emocional. Las nueve dimensiones del ERQS 
Tabla 3. Correlaciones entre las dimensiones del ERSQ y otros instrumentos, varianza media extraída

\begin{tabular}{|c|c|c|c|c|c|}
\hline & CERQ & DERS & SCL-90-R & AVE & $\sqrt{\mathrm{AVE}}$ \\
\hline Conciencia emocional & $.37 * * *$ & $-.43 * * *$ & $-.14 *$ & .78 & .88 \\
\hline Sensación & $.28 * *$ & $-.25 *$ & -.04 & .75 & .87 \\
\hline Claridad & $.33 * * *$ & $-.37 * * *$ & $-.27 * *$ & .73 & .85 \\
\hline Conocimiento & $.48 * * *$ & $-.58 * * *$ & $-.42 * * *$ & .69 & .83 \\
\hline Aceptación & $.35 * * *$ & $-.52 * * *$ & $-.23 * *$ & .72 & .85 \\
\hline Tolerancia & $.27 * *$ & $-.35 * * *$ & -.09 & .78 & .88 \\
\hline Disposición a confrontar & $.19 * *$ & $-.29 * *$ & $-.25 * *$ & .70 & .84 \\
\hline Auto-Ayuda & $.37 * * *$ & $-.38 * * *$ & $-.33 * * *$ & .74 & .86 \\
\hline Modificación & $.25 * *$ & $-.45 * * *$ & $-.19 *$ & .68 & .82 \\
\hline ERQS total & $.39 * * *$ & $-.52 * * *$ & $-.38 * * *$ & .72 & .85 \\
\hline
\end{tabular}

CERQ=Cognitive Emotion Regulation Questionnaire, DERS=Difficulties in Emotion Regulation Scale, SCL-90-R=Symptom Checklist 90 R $* p<.05 * * p<.01 * * * p<.001$

Tabla 4. Índices de bondad de ajuste

\begin{tabular}{lcccccc}
\hline Índice & $\mathrm{X}^{2}$ & $\mathrm{gl}$ & $\mathrm{CFI}$ & $\mathrm{TLI}$ & SRMR & RMSEA \\
\hline Modelo de dos factores & 1323.89 & 438 & .69 & .75 & .11 & .10 \\
Modelo de tres factores & 960.98 & 326 & .85 & .80 & .13 & .07 \\
Modelo de nueve factores & 752.72 & 310 & .97 & .95 & .05 & .04 \\
Criterio & & & $\geq .95$ & $\geq .95$ & $<.08$ & Cercanos a .06 \\
\hline
\end{tabular}

correlacionaron de manera significativa y positiva con el Cuestionario cognitivo de regulación emocional y, como era de esperarse, de manera negativa con la Escala de dificultades en la regulación emocional. Debido a que la Escala de dificultades en la regulación emocional mide las dificultades en el control de impulsos, el acceso limitado a estrategias de regulación, la falta de aceptación emocional, la interferencia en conductas dirigidas a metas, la falta de conciencia emocional y la falta de claridad emocional, se hipotetizó que tuviera una correlación negativa con las dimensiones y la puntuación total del ERSQ.

\section{Validez de constructo}

Finalmente, los datos obtenidos fueron sometidos a un análisis factorial confirmatorio empleando el método de estimación de máxima verosimilitud. Con base en la propuesta original del ERSQ, se diseñó un modelo de nueve factores con tres reactivos en cada uno. Este modelo se comparó con el modelo de tres factores encontrado en la versión en inglés y con el modelo de dos factores que se encontró en el análisis factorial exploratorio de esta investigación. Como se muestra en la Tabla 4, tanto el modelo de tres factores, como el modelo de dos factores reportaron un ajuste deficiente. Por el contrario, como también se documentó en la versión en inglés y la versión en japonés, en el modelo de nueve factores se obtuvieron resultados muy favorables en todos los indicadores. Como lo hacen constar los resultados obtenidos, los 27 reactivos quedaron agrupados en estos nueve factores mostrando la gran diversidad de estrategias de regulación emocional que mide el ERSQ. La versión final del instrumento puede ser consultada en el apéndice.

\section{Discusión}

Las pruebas psicométricas son un elemento fundamental en el análisis de las causas y los efectos de la regulación emocional. Los resultados de esta investigación contribuyen a proporcionar un instrumento en español de la regulación emocional. Al no contar con una versión en español del ERSQ, estamos contribuyendo a llenar este vacío en la literatura científica. Al igual que en su versión original, se pudo confirmar la estructura multidimensional del ERSQ. Los análisis llevados a cabo mostraron la presencia de nueve factores (Consciencia emocional, Sensación, Claridad, Conocimiento, Aceptación, Tolerancia, Disposición a confrontar, Auto-Ayuda y Modificación) midiendo diversas estrategias que se desarrollan para manejar las emociones. De esta manera, los resultados enfatizan la importancia de las habilidades que permiten el manejo adecuado de las emociones.

Siendo el objetivo de esta investigación analizar las propiedades psicométricas del ERSQ, los resultados revelaron una consistencia interna muy adecuada la cual osciló entre omega de McDonald=.65 (para la subescala de Disposición a confrontar hasta omega de McDonald=.94 para la puntuación total del cuestionario. Estos 
resultados son similares a los que se reportaron en la versión en inglés de la puntuación total del ERSQ $(\alpha=.96)$ (Grant et al., 2018). Con respecto a la fiabilidad test-retest llevada a cabo cuatro semanas después de la primera aplicación se obtuvieron coeficientes de correlación intraclase adecuados tanto para las nueve dimensiones como para la escala total $(\mathrm{ICC}=.88)$.

Con respecto a la distinción entre las estrategias de regulación emocional cognitivas y conductuales, esta quedó evidenciada en el conjunto de análisis que se llevaron a cabo para examinar la validez de constructo del ERSQ. Para la validez convergente se incluyeron dos de las pruebas que han reportado no solamente las mejores propiedades psicométricas, sino también un uso frecuente en múltiples investigaciones a nivel internacional. En este análisis se encontró que todas las correlaciones fueron estadísticamente significativas. Con respecto al Cuestionario cognitivo de regulación emocional, las correlaciones de mayor magnitud se presentaron con las dimensiones de Conocimiento $(r=.48)$, Consciencia emocional $(r=.37)$ y AutoAyuda ( $r=.37)$. A su vez, la Escala de dificultades en la regulación emocional presentó las correlaciones de mayor magnitud con el Conocimiento ( $r=-.58)$, Aceptación ( $r=-.52)$ y Modificación ( $r=-.45$ ). Por consiguiente, estos dos instrumentos presentaron las relaciones más importantes, mayoritariamente, con las estrategias cognitivas del ERSQ.

Por otra parte, para la validez divergente, los resultados evidenciaron que el ERSQ y el SCL90-R miden constructos diferentes. Específicamente, el conocimiento que se posee para identificar las emociones que se experimentan cotidianamente, así como las diversas acciones que lleva a cabo una persona para animarse ante emociones especialmente negativas tuvieron el mayor impacto en el nivel de distrés psicológico que presentaron los participantes. A la par, la escala en su totalidad tuvo también una de las correlaciones más significativas con el distrés psicológico. De la misma manera, en otros estudios previos se han encontrado relaciones negativas con otros constructos que evalúan deficiencias en la regulación emocional, diversas psicopatologías y disfunciones mentales (e.g., Berking et al., 2008;
Berking \& Znoj, 2008). Por consiguiente, en la presente investigación, tanto las estrategias cognitivas, como las conductuales mostraron los efectos que tienen las habilidades de regulación emocional en el distrés psicológico que experimenta una persona. Asimismo, los valores obtenidos en el índice AVE, la raíz cuadrada del AVE y la proporción HTMT mostraron evidencias con respecto a la adecuada validez discriminante del ERSQ.

Al analizar el uso de las estrategias de regulación emocional por parte de las mujeres y los hombres, encontramos que en términos generales las mujeres poseen una mejor regulación de sus emociones. Específicamente al comparar ambos sexos, las mujeres reportaron niveles más altos en la aceptación, claridad, conciencia emocional, disposición a confrontar, rumiación y reenfoque en la planeación, mientras que los hombres solamente mostraron un mayor nivel en el reenfoque positivo y auto-ayuda. Aunque en otros estudios llevados a cabo previamente en México se había identificado que tanto mujeres como hombres habían reportado niveles similares en el uso de las estrategias de reevaluación (Flores-Galaz et al., 2019) y en las de concentración, reflexión y cambio cognoscitivo (Sánchez-Aragón et al., 2014), en la presente investigación fueron las mujeres quienes mostraron un mayor manejo de sus emociones.

En el aspecto en el que coinciden los resultados de estos estudios, juntos con los encontrados en la presente investigación es en el predominio que tienen las estrategias cognitivas en lo referente al control de las emociones en la población mexicana. Particularmente en las familias mexicanas, los niños aprenden de sus padres por medio de la comunicación a desarrollar estas estrategias cognitivas que les permitirán en el futuro expresar y manejar adecuadamente sus emociones (Guaita, 2007). Una posible razón de este hallazgo es que los mexicanos de manera general tienden a priorizar los procesos cognitivos en comparación con los conductuales. De esta manera, en un primer momento se elaboran mentalmente una serie de estrategias encaminadas a dar una respuesta o solución apropiada a la situación que se presenta para posteriormente actuar en consecuencia. 
Una de las contribuciones más importantes de esta investigación fue la validación del ERSQ por medio de un análisis factorial confirmatorio. Los diferentes índices que se usaron muestran la bondad de ajuste del modelo confirmando con precisión la estructura multidimensional del Cuestionario. Estos índices coinciden con los encontrados en las versiones anteriores del ERSQ en inglés y japonés respaldando la validez del cuestionario. A diferencia de otros instrumentos que tienen una naturaleza unidimensional o que miden las estrategias de regulación emocional con dos, tres o cuatro factores, el ERSQ posee una amplia variedad de habilidades por medio de las cuales podemos tener una consciencia emocional de las experiencias y las conductas, sentir los efectos de las emociones en el cuerpo, tener claridad y conocer a profundidad las emociones, tolerar y aceptar aquello que no se puede cambiar, estar dispuesto a enfrentarse a situaciones difíciles, ser capaz de ayudarse a sí mismo y finalmente cambiar el impacto de las emociones negativas por medio de múltiples acciones cotidianas.

Con respecto a las limitaciones de esta investigación, en primer lugar, se puede señalar la falta de una representatividad de la muestra. Debido a que no se empleó un método probabilístico, no es posible asegurar que la muestra fue representativa de la población mexicana. Por otra parte, una segunda limitación recae en el diseño transversal lo cual impide que se puedan realizar inferencias de causalidad. Asimismo, el perfil sociodemográfico de la muestra es otra limitación. La mayoría de los participantes tienen un buen nivel educativo, tienen un trabajo estable y residen en una zona urbana. En futuros estudios sería recomendable incluir no solamente a participantes con distintas características sociodemográficas para tener una muestra más heterogénea, sino también a muestras clínicas. A su vez, al haber usado instrumentos de auto-informe es posible que se haya presentado cierta deseabilidad social lo cual pudo haber influido en las respuestas que emitieron los participantes. Finalmente, siendo el primer estudio que se ha llevado a cabo para adaptar y traducir el ERSQ al español, es necesario realizar más investigaciones para confirmar los resultados que se encontraron. A manera de conclusión, esta investigación confirma que el ERSQ posee las propiedades psicométricas apropiadas para su uso en población mexicana.

\section{Referencias}

Barrett, L. F., Lewis, M., \& Haviland-Jones, J. M. (2016). Handbook of Emotions (4th ed.). The Guilford Press.

Berke, D. S., Reidy, D. E., Gentile, B., \& Zeichner, A. (2019). Masculine discrepancy stress, emotion-regulation difficulties and intimate partner violence. Journal of Interpersonal Violence, 34(6), 1163-1182.

Berking, M. (2008). Training emotionaler Kompetenzen. Heidelberg: Springer-Verlag.

Berking, M., Meier, C., \& Wupperman, P. (2010). Enhancing emotion-regulation skills in police officers: Results of a pilot controlled study. Behavior Therapy, 41(3), 329-339.

Berking, M., \& Schwarz, J. (2014). Affect regulation training. In J. J. Gross (Ed.), Handbook of emotion regulation (2nd ed.) (pp. 529-547). Guilford Press.

Berking, M., \& Whitley, B. (2014). Affect Regulation Training. Springer.

Berking, M., Wupperman, P., Reichardt, A., Pejic, T., Dippel, A., \& Znoj, H. (2008). General emotion-regulation skills as a treatment target in psychotherapy. Behaviour Research and Therapy, 46, 1230-1237.

Berking, M., \& Znoj, H. (2008). Entwicklung und Validierung eines Fragebogens zur standardisierten Selbsteinschätzung emotionaler Kompetenzen (SEK-27) [Development and validation of the Emotion Regulation Skills Questionnaire (ERSQ-27)]. Zeitschrift für Psychiatrie, Psychologie und Psychotherapie, 52, 142-151.

Cavelti, M., Corbisiero, S., Bitto, H., Moerstedt, B., Newark, P., Faschina, S., ... \& Stieglitz, R. D. (2019). A comparison of self-reported emotional regulation skills in adults with attention-deficit/hyperactivity disorder and borderline personality disorder. Journal of Attention Disorders, 23(12), 1396-1406.

Cruz-Fuentes, C. S., López-Bello, L., Blas-García, C., González-Macías, L., \& Chávez-Balderas, R. A. (2005). Datos sobre la validez y 
confiabilidad de la Symptom Check List 90 (SCL 90) en una muestra de sujetos mexicanos. Salud Mental, 28(1), 72-81.

Derogatis, L. (1994). SCL-90-R. Symptom Checklist-90-R. Administration, scoring and procedures manual. National Computer System.

Diedrich, A., Burger, J., Kirchner, M., \& Berking, M. (2017). Adaptive emotion regulation mediates the relationship between selfcompassion and depression in individuals with unipolar depression. Psychology and Psychotherapy: Theory, Research and Practice, 90(3), 247-263. http://dx.doi.org/10.1111/papt.12107

Domínguez-Sánchez, F. J., Lasa-Aristu, A., Amor, P. J., \& Holgado-Tello, F. P. (2013). Psychometric properties of the Spanish version of the Cognitive Emotion Regulation Questionnaire. Assessment, 20(2), 253-261. http://dx.doi.org/10.1177/ 1073191110397274

Eckert, M., Ebert, D. D., Lehr, D., Sieland, B., \& Berking, M. (2016). Overcome procrastination: Enhancing emotion regulation skills reduce procrastination. Learning and Individual Differences, 52, 10-18. http://dx.doi.org/10.1016/ j.lindif.2016.10.001

Eisenberg, N., \& Spinrad, T. L. (2004). Emotionrelated regulation: Sharpening the definition. Child Development, 75, 334-339. http://dx.doi.org/10.1111/j.14678624.2004.00674.x

Flora, D. B. (2020). Your coefficient alpha is probably wrong, but which coefficient omega is right? A tutorial on using $\mathrm{R}$ to obtain better reliability estimates. Advances in Methods and Practices in Psychological Science, 3(4), 484501.

Flores-Galaz, M. M., Morales, M. T., Cortes, M. L., Campos, M. J., \& Díaz-Loving, R. (2019). Crianza materna y regulación emocional en adolescentes: Diferencias asociadas al sexo. Emerging Trends in Education, 1(2), 96-120. http://dx.doi.org/ 10.19136/etie.a1n2.2781

Fornell, C., \& Larcker, D. F. (1981). Evaluating structural equations models with Unobservable variables and measurement error. Journal of Marketing Research, 18(1), 39-50.
Fujisato, H., Ito, M., Takebayashi, Y., Hosogoshi, H., Kato, N., Nakajima, S., ... \& Horikoshi, M. (2017). Reliability and validity of the Japanese version of the Emotion Regulation Skills Questionnaire. Journal of Affective Disorders, 208, 145-152.

http://dx.doi.org/10.1016/j.jad.2016.08.064

Garnefski, N., Kraaij, V., \& Spinhoven, P. (2001). Negative life events, cognitive emotion regulation, and emotional problems. Personality and Individual Differences, 30, 1311-1327.

http://dx.doi.org/10.1016/S01918869(00)00113-6

Garnefski, N., \& Kraaij, V. (2007). The Cognitive Emotion Regulation Questionnaire. Psychometric features and prospective relationships with depression and anxiety in adults. European Journal of Psychological Assessment, 23, 141-149. http://dx.doi.org/10.1027/1015-5759.23.3.141

George, D., \& Mallery, P. (2013). IBM SPSS Statistics 21 step by step: A simple guide and reference (13th ed.). Pearson.

Grant, M., Salsman, N. L., \& Berking, M. (2018). The assessment of successful emotion regulation skills use: Development and validation of an English

version of the Emotion Regulation Skills Questionnaire. PLOS ONE 13(10): e0205095. http://dx.doi.org/10.1371/journal.pone.0205095

Gratz, K. L., \& Roemer, E. (2004). Multidimensional assessment of emotion regulation and dysregulation: Development, factor structure and initial validation of the difficulties in Emotion Regulation Scale. Journal of Psychopathology and Behavioral Assessment, 26(1), 41-54. http://dx.doi.org/10.1007/s10862-008-9102-4

Gross, J. J. (1998). The emerging field of emotion regulation: An integrative review. Review of General Psychology, 2, 271-299. http://dx.doi.org/10.1037/1089-2680.2.3.271

Gross, J. J. (1999). Emotion regulation: Past, present, future. Cognition and Emotion, 13, 551-573.

https://doi.org/10.1080/026999399379186 
Gross, J., \& John, O. (2003). Individual differences in two emotion regulation processes: Implications for affect, relationships, and well-being. Journal of Personality and Social Psychology, 85(2), 348-362. http://dx.doi.org/10.1037/0022-3514.85.2.348

Guaita, V. L. (2007). Aspectos emocionales de la comunicación en niños en riesgo por pobreza extrema. Revista Iberoamericana de Diagnóstico y Evaluación - e Avaliação Psicológica, 2(24), 177-192.

Henseler, J., Ringle, C. M., \& Sarstedt, M. (2015). A new criterion for assessing discriminant validity in variance-based structural equation modelling. Journal of the Academy of Marketing Science, 43(1), 115-135. https://doi.org/10.1007/s11747-014-0403-8

Hervás, G. (2011). Psicopatología de la regulación emocional: El papel de los déficit mocionales en los trastornos clínicos. Psicología Conductual, 19, 347-372.

Hu, L., \& Bentler, P. M. (1999). Cutoff criteria for fit indexes in covariance structure analysis: Conventional criteria versus new alternatives. Structural Equation Modeling, 6, 1-55. http://dx.doi.org/10.1080/10705519909540118

Jöreskog, K. G., \& Sörbom, D. (1996). LISREL 8: User's reference guide. Chicago: Scientific Software International.

Kline, R. B. (2011). Principles and Practice of Structural Equation Modeling ( $3^{\circ} \mathrm{ed}$.). The Guilford Press.

Koo, T. K., \& Li, M. Y. (2016). A Guideline of selecting and reporting intraclass correlation coefficients for reliability research. Journal of Chiropractic Medicine,15, 155-163. http://dx.doi.org/10.1016/j.jcm.2016.02.012

Lawlor, C., Hepworth, C., Smallwood, J., Carter, B., \& Jolley, S. (2020). Self-reported emotion regulation difficulties in people with psychosis compared with non-clinical controls: A systematic literature review. Clinical Psychology \& Psychotherapy, 27(2), 107-135.

Lincoln, T. M., Hartmann, M., Köther, U., \& Moritz, S. (2015). Dealing with feeling: Specific emotion regulation skills predict responses to stress in psychosis. Psychiatry Research, 228(2), 216-222. http://dx.doi.org/10.1016/

j.psychres.2015.04.003

Lukas, C. A., Ebert, D. D., Fuentes, H. T., Caspar, F., \& Berking, M. (2018). Deficits in general emotion regulation skills - Evidence of a transdiagnostic factor. Journal of Clinical Psychology, 74(6), 1017-1033.

Medrano, L. A., Moretti, L., Ortiz, Á., \& Pereno, G. (2013). Validación del cuestionario de regulación emocional cognitiva en universitarios de Córdoba, Argentina. Psykhe, 22(1), 83-96.

Moradi-Siahafshadi, M., Amiri, S., Molavi, H., \& Ghasemi, N. (2018). The relationship between attachment and subjective well-being: The mediating role of emotion regulation skill. International Journal of Psychology, 12(1), 118-135.

http://dx.doi.org/10.24200/ijpb.2018.58150

Netemeyer, R. G., Johnston, M. W., \& Burton, S. (1990). Analysis of role conflict and role ambiguity in a structural equations framework. Journal of Applied Psychology, 75(2), 148-157.

Owens, G. P., Held, P., Hamrick, L., \& Keller, E. (2018). The indirect effects of emotion regulation on the association between attachment style, depression and meaning made among undergraduates who experienced stressful events. Motivation and Emotion, 42(3), 429-437.

Pocnet, C., Dupuis, M., Congard, A., \& Jopp, D. (2017). Personality and its links to quality of life: Mediating effects of emotion regulation and self-efficacy beliefs. Motivation and Emotion, 41(2), 196-208.

$\mathrm{R}$ Core Team (2020). R: A language and environment for statistical computing. $\mathrm{R}$ Foundation for Statistical Computing, Vienna, Austria. https://www.R-project.org/."

Salessi, S., \& Omar, A. (2016). Desarrollo y validación de una escala para medir actuación emocional en el trabajo. Revista Iberoamericana de Diagnóstico y Evaluación - e Avaliação Psicológica, 1(41), 66-79.

Sánchez-Aragón, R., Jiménez-Rodríguez, B., \& Perales-González, V. (2014). Medición y contrastes en mecanismos regulatorios de la tristeza en hombres y mujeres. Psicología y Salud, 24(2), 221-234. 
Schmitz, N., Kruse, J., Heckrath, C., Alberti, L., \& Tress, W. (1999). Diagnosing mental disorders in primary care: The General Health Questionnaire [GHQ] and the Symptom Checklist [SCL-90-R] as screening instruments. Social Psychiatry and Psychiatric Epidemiology, 34(7), 360-366. http://dx.doi.org/10.1007/s001270050156

Schumacker, R. E., \& Lomax, R. G. (2016). A beginner's guide to structural equation modeling (4th ed.). Routledge.

Shields, A., \& Cicchetti, D. (1997). Emotion regulation among school-age children: The development and validation of a new criterion Q-sort scales. Developmental Psychology, 33, 906-916. http://dx.doi.org/10.1037/00121649.33.6.906

Shriver, L. H., Dollar, J. M., Calkins, S. D., Keane, S. P., Shanahan, L., \& Wideman, L. (2021). Emotional eating in adolescence: Effects of emotion regulation, weight status and negative body image. Nutrients, 13(1), 79.

Shrout, P. E., \& Fleiss, J. L. (1979). Intraclass correlations: Uses in assessing rater reliability. Psychological Bulletin, 86, 420-428. ttp://dx.doi.org/10.1037/0033-2909.86.2.420

Zhang, J., Lau, E. Y. Y., \& Hsiao, J. H. (2019). Using emotion regulation strategies after sleep deprivation: ERP and behavioral findings. Cognitive, Affective, \& Behavioral Neuroscience, 19, 283-295. 


\section{Apéndice}

\section{Cuestionario de Habilidades de Regulación Emocional (Emotion-Regulation Skills Questionnaire)}

Instrucciones: A continuación se presenta una lista con enunciados que describen la manera en que las personas regulan sus emociones. Indique por favor con una X el grado de acuerdo con cada una de ellas utilizando la siguiente escala del 0 al 4.

0

No del todo
1

Pocas veces
2

Algunas veces
3

Frecuentemente
4

Casi siempre

1 Fui capaz de poner atención de manera consciente a mis sentimientos.

2 Pude generar conscientemente sentimientos positivos.

3 Entendí mis reacciones emocionales.

4 Pude superar mis sentimientos negativos.

5 Fui capaz de aceptar mis sentimientos negativos

6 Pude darles un nombre a mis sentimientos.

7 Tuve una clara percepción de los efectos físicos que tienen mis sentimientos.

8 Hice lo que yo quería hacer incluso si tenía que enfrentar sentimientos negativos como consecuencia de ello.

9 Traté de tranquilizarme a mí mismo durante situaciones de angustia.

10 Fui capaz de influir en mis sentimientos negativos.

11 Supe lo que mis sentimientos significaban para mí.

12 Pude enfocarme en mis emociones negativas cuando era necesario.

13 Supe identificar las emociones que estaba sintiendo en ese momento.

14 Me di cuenta conscientemente cuando mi cuerpo reaccionó de una forma específica ante situaciones emocionales.

15 Traté de animarme durante situaciones de angustia emocional.

16 Hice lo que yo quería hacer a pesar de mis sentimientos negativos.

17 Estuve bien con mis sentimientos incluso cuando eran negativos.

18 Estaba seguro de que podría tolerar incluso sentimientos intensamente negativos.

19 Fui capaz de experimentar conscientemente mis sentimientos.

20 Fui consciente del por qué me sentía de la manera en que me sentía.

21 Supe que era capaz de influir en mis sentimientos.

22 Perseguí metas que eran importantes para mí, incluso al pensar que al hacerlo desencadenaría o intensificaría sentimientos negativos.

23 Fui capaz de experimentar mis sentimientos negativos sin tratar inmediatamente de luchar contra ellos.

24 Mis sensaciones físicas fueron un buen indicador de cómo me estaba sintiendo.

25 Tuve la suficiente claridad con respecto a las emociones que estaba experimentando.

26 Pude tolerar mis sentimientos negativos.

27 Me ayudé a mí mismo durante situaciones de angustia emocional. 